\title{
An aide mémoire: working on acute medical assessment units
}

\author{
Naseem Naqvi*
}

Acute medicine is an emerging speciality that has now been approved by most postgraduate education and training boards as a distinct branch of medicine concerned with the immediate and early management of acutely ill patients. Acute medical assessment units are an interface between emergency medicine and inpatient care, and are the front door of hospitals. Working on these units is exciting as well as challenging. With my background of working on these units in National Health Service in the UK, readers, especially junior doctors, will find the article useful if they are intending to pursue a career in acute medicine.

Acute medical assessment units have rapid turn-over of patients; it is essential for staff and doctors working on these units to manage their time effectively, prioritise clinical needs, and learn to multitask to avoid redundancy and duplication of work. It is also vital to acknowledge and appreciate the contribution of your colleagues to boost up their morale and team spirit.

Information handling is an important area of acute care which is often mismanaged. Work loads and time constraints often prevent us from harmonizing in patient care. Firstly, it is essential to be fully aware of the trust protocols and local guidelines. It is always helpful to search for old case notes and to look up electronic patient case records if available. One should not hesitate to ring up the GP or nursing homes, as we may sometimes get valuable information which may not be readily available from patients, especially if they are demented, blind or deaf. Furthermore, when encountering a language barrier with a patient, it is worth considering the Patient Language Help Line (NHS Language line in UK).

\footnotetext{
*To whom correspondence should be addressed:

Naseem Naqvi MBBS, MRCP1(Edin), Senior House Officer Acute Medicine, Wrightington, Wigan \& Leigh NHS Trust, Member Society For Acute Medicine UK Tel: +441942340508

Email: naseemnaqvi@doctors.org.uk
}

Since acute medicine has been introduced as a distinct discipline, there are concerns regarding the education, training, career pathways, and job plans of doctors who are drawn to this field, as well as the need for an academic base to ensure teaching and research of the highest quality. In most countries including the UK, curriculum will run from Foundation years to acquisition of CCST (certificate of completion of specialist training). For most junior doctors rotating through various medical specialities during their $\mathrm{SHO} /$ Residency years, a work experience on acute admissions units is their only opportunity to learn and practice acute general medicine. Also, these are the best places to learn practical procedural skills: central venous catheter line placement, intercostal drain placement, lumbar punctures, temporary pacings etc. An expertise in these procedures is an essential skill in the armoury of a physician intending to pursue his career in acute medicine.

Acute health care is complex to manage; patients now seek assurance that doctors remain safe throughout their practicing lives and rising expectations are a mark of our success. The concept of safety-netting holds its vital importance when it comes to working on acute medical assessment units. It is imperative that one should know and realize his/her limitations and ask for a senior's help when one feels out of breadth. We must not hesitate to contact the consultant or Senior Registrar on call if any doubts regarding the management plan. Also, it is worth contacting specialist centres for advice if you work in a small district general hospital.

There has been much activity and growth in acute medicine as a speciality. For acute physicians, the challenge over the last decade has been to develop strategies to cope with the increasing demands of accountability in acute care. While on the other hand, there is a constant need to examine the education and training of healthcare professionals to ensure the delivery of patient-focused quality of care. 


\section{Aide Mémoire: important points to keep in mind}

- Make sure that the medical staffing and human resources are aware of your on-call swaps

- Page yourself before starting on-call duty to double check that your pager is working

- Try to present as many cases as possible to the on-call consultant; it will improve your case summarising skills, which are invaluable for passing clinical examinations.

- Always look for any potentially treatable cause of confusion in the elderly before labelling them as demented

- The medical assessment unit is probably the best place to consolidate your communication skills with the patients and relatives, and to involve them at every stage of the management plan.

- Try not to discuss about patients and their illness when having your cup of tea during your breaks

- Think twice before sending a patient home from the medical assessment unit. Verify that you are not missing any serious underlying disease. If unsure, detain and always asks yourself: "what if?"

- Keep a diary/PDA with you to make note of any interesting cases or rare disease you have seen.

- Make every effort to publish rare and/or interesting casse in the case reports section of medical journals. It will be a positive addition to your $\mathrm{CV}$ and will help when you start applying for sought-after senior grade career positions

- The diagnosis often comes out of the patient's mouth; listen to them and focus on non-verbal clues

- Always think of common and potentially treatable illnesses when considering a diagnosis, but remember: rare is rare, but rare is always there

Naseem Naqvi (MBBS, MRCP 2) is second-year speciality trainee in acute medicine at the Aintree University Hospitals NHS Trust in the UK and a member of the Society for Acute Medicine. 